\title{
Quasi-Sliding Networked Control of Systems Subject to Unbounded Disturbance with Limited Rate of Change
}

\author{
Andrzej Bartoszewicz and Paweł Latosiński \\ Institute of Automatic Control, Technical University of Łódź, 18/22 Stefanowskiego Street, 90-924 Łódź, Poland \\ Correspondence should be addressed to Andrzej Bartoszewicz; andrzej.bartoszewicz@p.lodz.pl
}

Received 24 July 2014; Revised 29 October 2014; Accepted 30 October 2014

Academic Editor: Rongni Yang

Copyright ( 2015 A. Bartoszewicz and P. Latosiński. This is an open access article distributed under the Creative Commons Attribution License, which permits unrestricted use, distribution, and reproduction in any medium, provided the original work is properly cited.

\begin{abstract}
This paper concerns network based sliding mode control of linear plants with state measurement delay. The considered plants are subject to unbounded disturbance, but it is assumed that the change of disturbance value between each two subsequent sampling instants is limited. In order to combat the unpredictable disturbance in the environment with state measurement delay, a novel sliding mode controller has been introduced. It utilizes two nominal models of the plant to drive the system state along a desired trajectory and counteract the predicted effect of the past disturbance on the system. It has been proven that applying the new control strategy to the plant confines the system state to a defined band around the sliding hyperplane.
\end{abstract}

\section{Introduction}

Continuous-time variable structure control (VSC) has gained a considerable popularity in control engineering community since its introduction in the early 1960 s $[1,2]$. Its main advantages are computational efficiency and insensitivity with respect to matched disturbance [3]. These attractive properties resulted in a considerable amount of research dedicated to sliding mode control, which is the most widely used method of VSC implementation [4-8]. In mid-1980s, the theory of variable structure control has been extended to discrete-time systems $[9,10]$, which in turn inspired many significant works related to discrete-time sliding mode control [11-33]. In particular, discrete-time sliding mode control has great significance in digital environments [34,35], where no continuous states are available.

Since the introduction of relatively low-cost control networks [36-41], the problem of effectively controlling discretetime systems with time delay [42-46] gained importance, as the time it takes for the sensor data and control signal to travel through the network is often nonnegligible. With this in mind, we will design an effective sliding mode control strategy for discrete-time systems with time delay. As opposed to the control method proposed in our previous paper [47], the new approach will realize trajectory tracking in a networked environment as well as ensuring upper bounded convergence rate to the vicinity of the switching hyperplane. To that end, we will introduce two nominal models of the controlled plant. One of them will be compared to the original plant in order to extract delayed information about disturbance affecting the system. Then, the current effect of the disturbance on the system will be estimated and reduced to zero with a dead-beat controller. The other model will be utilized to drive the system from its initial state to a desired one in finite time and realize trajectory tracking in subsequent time instants.

Typically, in the design process of a sliding mode controller, an upper bound of the disturbance affecting the controlled plant is assumed to be known [14, 16, 21, 47]. However, in this paper we will abandon that assumption in favor of the one proposed in [15]. In other words, we assume that the change of the disturbance value between every two subsequent time instants is bounded by a certain constant $\Delta_{\max }$, while the value of the disturbance itself does not have to be bounded. Under this assumption, we will design a sliding mode controller that will effectively combat the effect of the disturbance on the system in a networked environment. 
The remainder of this paper is organized in the following way. Section 2 states the considered problem, after which the new control strategy is introduced in Section 3. The control law for the considered system is established and the properties of the controlled system are formally proven in the same section. In Section 4, two simulation examples that show the effectiveness of the proposed method are presented and concluding remarks are given in Section 5.

\section{Problem Statement}

Let us consider a single input single output, linear, time invariant plant:

$$
\mathbf{x}(k+1)=\mathbf{A x}(k)+\mathbf{b} u(k)+\mathbf{b} d(k),
$$

where $\mathbf{A}$ is the state matrix such that $\operatorname{dim} \mathbf{A}=n \times n, \mathbf{b}$ and $\mathbf{x}(k)$ are vectors of appropriate dimensions that represent input distribution and system state, respectively, and $u(k)$ and $d(k)$ are scalars that represent the control signal and disturbance, respectively. We assume that the plant operates in a networked environment, which means that it is subject to network-induced delay $\alpha$. Therefore, the control signal $u(k)$ in each time instant $k$ is calculated based on the system states up to the moment $k-\alpha$. We do not assume that the disturbance affecting the system is bounded, but that the change of the disturbance value between every two subsequent time instants is limited by $\Delta_{\max }$. In other words, $|d(k)-d(k-1)| \leq \Delta_{\max }$ for any $k=1,2,3, \ldots$

\section{Proposed Control Strategy}

We begin by introducing two models of the considered system (1). The first one

$$
\mathbf{y}(k+1)=\mathbf{A y}(k)+\mathbf{b} u(k)
$$

will be used to extract information about disturbance from the system. The matrix $\mathbf{A}$ and vector $\mathbf{b}$ in the model are the same as in the original system and $\mathbf{y}(0)=\mathbf{x}(0)$. The model is controlled with the same signal $u(k)$ as plant (1). Moreover, let us define another model

$$
\mathbf{z}(k+1)=\mathbf{A z}(k)+\mathbf{b} u_{0}(k)
$$

controlled with a different signal $u_{0}$. Again, $\mathbf{A}$ and $\mathbf{b}$ are the same as in (1) and $\mathbf{z}(0)=\mathbf{x}(0)$. The second model will be used solely to drive the system along the desired trajectory $\mathbf{x}_{d}(k)$. We take into account the sliding hyperplane defined as

$$
s(k)=\mathbf{c}^{\mathrm{T}} \mathbf{e}(k)=0
$$

where $\mathbf{e}(k)=\mathbf{x}_{d}(k)-\mathbf{x}(k)$ and $\mathbf{c}$ is such a vector that

$$
\operatorname{det}\left[\lambda \mathbf{I}_{n}-\mathbf{A}+\mathbf{b}\left(\mathbf{c}^{\mathbf{T}} \mathbf{b}\right)^{-1} \mathbf{c}^{\mathbf{T}} \mathbf{A}\right]=\lambda^{n} .
$$

It can be seen that vector c would allow us to design a dead-beat controller for the delay-free system. Although the existing delay prevents us from applying such a controller to the system (1), vector $\mathrm{c}$ is chosen in such a way that allows us to compensate for the effect of past disturbances on the system as well as partially reducing the ones that have not yet been estimated due to the delay. We first define the following sequence of $n$ dimensional vectors:

$$
\begin{aligned}
\mathbf{w}(k)= & {[\mathbf{x}(k-\alpha)-\mathbf{A} \mathbf{x}(k-\alpha-1)] } \\
& -[\mathbf{y}(k-\alpha)-\mathbf{A y}(k-\alpha-1)]
\end{aligned}
$$

that will be used to extract the information about past disturbances from the system. We assume that $\mathbf{x}(k)=\mathbf{y}(k)=$ $\mathbf{0}$ for any $k<0$, which gives $\mathbf{w}(k)=\mathbf{0}$ for $k \leq \alpha$. For any $k>\alpha$,

$$
\begin{aligned}
\mathbf{w}(k)= & {[\mathbf{x}(k-\alpha)-\mathbf{A} \mathbf{x}(k-\alpha-1)] } \\
& -[\mathbf{y}(k-\alpha)-\mathbf{A} \mathbf{y}(k-\alpha-1)] \\
= & {[\mathbf{A x}(k-\alpha-1)+\mathbf{b} u(k-\alpha-1)} \\
& +\mathbf{b} d(k-\alpha-1)-\mathbf{A} \mathbf{x}(k-\alpha-1)] \\
& -[\mathbf{A y}(k-\alpha-1)-\mathbf{b} u(k-\alpha-1)-\mathbf{A y}(k-\alpha-1)] \\
= & \mathbf{b} d(k-\alpha-1) .
\end{aligned}
$$

Therefore, at any time instant $k$, we have

$$
\mathbf{w}(k)= \begin{cases}0 & \text { for } k \leq \alpha \\ \mathbf{b} d(k-\alpha-1) & \text { for } k>\alpha .\end{cases}
$$

We will now design a control law that combats the effect of the disturbance obtained from (8) on the system and partially reduces the effect of $\alpha$ subsequent disturbances that have not yet been explicitly obtained due to the delay $\alpha$. This is possible since the disturbance rate of change is limited. Let us define $n+1$ vectors $\mathbf{v}_{1}(k), \ldots, \mathbf{v}_{n+1}(k)$ in the following way:

$$
\begin{gathered}
\forall_{i \in\{1, \ldots, n+1\}} \forall_{k<i} \quad \mathbf{v}_{i}(k)=0 \\
\forall_{k \geq 1} \quad \mathbf{v}_{1}(k)=\sum_{i=0}^{\alpha} \mathbf{A}^{i}[\mathbf{w}(k)-\mathbf{w}(k-1)] \\
=\sum_{i=0}^{\alpha} \mathbf{A}^{i} \mathbf{b}[d(k-\alpha-1)-d(k-\alpha-2)] \\
\forall_{i \in\{2, \ldots, n+1\}} \forall_{k \geq i} \quad \mathbf{v}_{i}(k)=\mathbf{A} \mathbf{v}_{i-1}(k-1)+\mathbf{b} u_{i-1}(k-1),
\end{gathered}
$$

where

$$
\forall_{i \in\{1, \ldots, n\}} \forall_{k} \quad u_{i}(k)=-\left(\mathbf{c}^{\mathrm{T}} \mathbf{b}\right)^{-1} \mathbf{c}^{\mathrm{T}} \mathbf{A v}_{i}(k)
$$

It can be seen from (9) that each vector $\mathbf{v}_{i}$ can be expressed as

$$
\begin{aligned}
\forall_{i \in\{2, \ldots, n+1\}} \quad \mathbf{v}_{i}(k)= & \mathbf{A}^{i-1} \mathbf{v}_{1}(k-i+1) \\
& +\sum_{j=0}^{i-2} \mathbf{A}^{j} \mathbf{b} u_{i-j-1}(k-j-1) .
\end{aligned}
$$

Vectors $\mathbf{v}_{i}(k)$ possess an important property described by Lemma 1. 
Lemma 1. For any $k$, the vector $\mathbf{v}_{n+1}(k)=\mathbf{0}$. Furthermore, for $i=2, \ldots, n$, the product $\mathbf{c}^{\mathrm{T}} \mathbf{v}_{i}(k)=0$.

Proof. The proof of this property is given in our previous work [47].

The control laws (10) will be used to reduce each individual disturbance value affecting the system (1) to zero in finite time. This property will be demonstrated in Theorem 2 later in this paper. We will now define the control law $u_{0}$ that will drive the system from its initial position $\mathbf{x}(0)$ to a desired trajectory $\mathbf{x}_{d}(k)$. Let $s_{z}(k)=\mathbf{c}^{\mathbf{T}}\left[\mathbf{x}_{d}(k)-\mathbf{z}(k)\right]$ and let us consider the reaching law [15]:

$$
s_{z}(k+1)=\frac{k^{*}-k}{k^{*}} s_{z}(k)
$$

where $k^{*}$ is a natural number chosen by the designer. It can be seen that the reaching law designed in such a way guarantees that the reference sliding variable $s_{z}(k)$ will be reduced to 0 at the moment $k^{*}+1$ and will retain that value in every subsequent step. The control law $u_{0}(k)$ for model (3) obtained from reaching law (12) can be expressed as

$$
\begin{aligned}
u_{0}(k)= & \left(\mathbf{c}^{\mathrm{T}} \mathbf{b}\right)^{-1} \mathbf{c}^{\mathrm{T}} \\
& \times\left[\mathbf{x}_{d}(k+1)-\mathbf{A z}(k)-\frac{k^{*}-k}{k^{*}}\left(\mathbf{x}_{d}(k)-\mathbf{z}(k)\right)\right] .
\end{aligned}
$$

We propose the following control law for system (1):

$$
\begin{aligned}
u(k)= & u_{0}(k)+u_{1}(k)+u_{2}(k)+\cdots+u_{n}(k) \\
& -\left(\mathbf{c}^{\mathrm{T}} \mathbf{b}\right)^{-1} \mathbf{c}^{\mathrm{T}} \mathbf{w}(k),
\end{aligned}
$$

where $u_{0}$ is defined by (13), $u_{1}, \ldots, u_{n}$ are defined by (10), and $\mathbf{w}$ is defined by (8). Control law designed in such a way will drive the system from its initial position to a desired state $\mathbf{x}_{d}$ in a finite amount of steps, combat the effect of disturbances up to the moment $k-\alpha$ on the sliding variable, and partially reduce the effects of disturbances that occurred after $k-\alpha$. Additionally, the element $-\left(\mathbf{c}^{\mathrm{T}} \mathbf{b}\right)^{-1} \mathbf{c}^{\mathrm{T}} \mathbf{w}(k)$ will partially counteract the disturbance $d(k)$ that occurs at the moment of applying the control law. As a result, the system state will be confined to a certain band around the sliding hyperplane. The width of the band is specified by Theorem 2 formulated below.

Theorem 2. If the control signal $u(k)$ for system (1) is defined by (14) and the system is subject to disturbance $d(k)$, which satisfies $|d(k)-d(k-1)| \leq \Delta_{\max }$ for any $k>0$, then there exists a natural number $k_{0}$ such that for every $k>k_{0}$ the system state is confined to a quasi-sliding mode band defined as

$$
|s(k)|=\left|\mathbf{c}^{\mathbf{T}}\left[\mathbf{x}_{d}-\mathbf{x}(k)\right]\right| \leq \Delta_{\max } \cdot \sum_{i=0}^{\alpha}(i+1)\left|\mathbf{c}^{\mathbf{T}} \mathbf{A}^{\alpha-i} \mathbf{b}\right|
$$

Proof. Let $k>\max \left\{\alpha+n, k^{*}\right\}$. First, we express $\mathbf{x}(k+1)$ as

$$
\begin{aligned}
\mathbf{x}(k+1)= & \mathbf{A} \mathbf{x}(k)+\mathbf{b} u(k)+\mathbf{b} d(k) \\
= & \mathbf{A}^{2} \mathbf{x}(k-1)+\mathbf{A} \mathbf{b} u(k-1)+\mathbf{A} \mathbf{b} d(k-1) \\
& +\mathbf{b} u(k)+\mathbf{b} d(k) \\
= & \cdots=\mathbf{A}^{k+1} \mathbf{x}(0)+\sum_{m=0}^{k} \mathbf{A}^{k-m} \mathbf{b} u(m) \\
& +\sum_{m=0}^{k} \mathbf{A}^{k-m} \mathbf{b} d(m) .
\end{aligned}
$$

We rewrite each disturbance $d(i)$ as $d\left(i-\gamma_{i}\right)+d\left(i-\gamma_{i}+1\right)-$ $d\left(i-\gamma_{i}\right)+\cdots+d(i)-d(i-1)$, where $\gamma_{i}=\min \{i, \alpha+1\}$. We obtain

$$
\begin{aligned}
\mathbf{x}(k+1) & \\
= & \mathbf{A}^{k+1} \mathbf{x}(0)+\sum_{m=0}^{k} \mathbf{A}^{k-m} \mathbf{b} u(m) \\
& +\mathbf{A}^{k} \mathbf{b} d(0)+\mathbf{A}^{k-1} \mathbf{b}[d(0)+d(1)-d(0)] \\
& +\mathbf{A}^{k-2} \mathbf{b}[d(0)+d(1)-d(0)+d(2)-d(1)] \\
& +\cdots+\mathbf{A}^{k-\alpha} \mathbf{b} \\
& \quad \times[d(0)+d(1)-d(0)+\cdots+d(\alpha)-d(\alpha-1)] \\
& +\mathbf{A}^{k-\alpha-1} \mathbf{b} \\
& \times[d(0)+d(1)-d(0)+\cdots+d(\alpha+1)-d(\alpha)] \\
& +\mathbf{A}^{k-\alpha-2} \mathbf{b} \\
& \times[d(1)+d(2)-d(1)+\cdots+d(\alpha+2)-d(\alpha+1)] \\
& +\cdots+\mathbf{A}^{k-\alpha-3} \mathbf{b} \\
& \times[d(2)+d(3)-d(2)+\cdots+d(\alpha+3)-d(\alpha+2)] \\
+ & \cdots+\mathbf{b} \\
& \times[d(k-\alpha-1)+d(k-\alpha)-d(k-\alpha-1)
\end{aligned}
$$

We now rearrange the elements in (17) in the following way. For every $i<k-\alpha, n+1$ elements containing $[d(i)-d(i-$ $1)$ ] multiplied by a different power of $\mathbf{A}$ are grouped together. 
Then, in each group, the highest power of $\mathbf{A}$ is factored out. In this way we get

$$
\begin{aligned}
\mathbf{x} & +1) \\
= & \mathbf{A}^{k+1} \mathbf{x}(0)+\sum_{m=0}^{k} \mathbf{A}^{k-m} \mathbf{b} u(m)+\sum_{m=0}^{k-\alpha-1} \mathbf{A}^{m} \mathbf{b} d(k-\alpha-1-m) \\
& +\mathbf{A}^{k-\alpha} \sum_{i=0}^{\alpha} \mathbf{A}^{i} \cdot \mathbf{b} d(0)+\mathbf{A}^{k-\alpha-1} \sum_{i=0}^{\alpha} \mathbf{A}^{i} \cdot \mathbf{b}[d(1)-d(0)] \\
& +\cdots+\mathbf{A}^{n} \sum_{i=0}^{\alpha} \mathbf{A}^{i} \cdot \mathbf{b}[d(k-n-\alpha)-d(k-n-\alpha-1)] \\
& +\mathbf{A}^{n-1} \sum_{i=0}^{\alpha} \mathbf{A}^{i} \cdot \mathbf{b}[d(k-n-\alpha+1)-d(k-n-\alpha)] \\
& +\cdots+\mathbf{A}^{2} \sum_{i=0}^{\alpha} \mathbf{A}^{i} \cdot \mathbf{b}[d(k-\alpha-2)-d(k-n-\alpha-3)] \\
& +\mathbf{A} \sum_{i=0}^{\alpha} \mathbf{A}^{i} \cdot \mathbf{b}[d(k-\alpha-1)-d(k-\alpha-2)] \\
& +\mathbf{A}^{\alpha} \mathbf{b}[d(k-\alpha)-d(k-\alpha-1)] \\
& +\mathbf{A}^{\alpha-1} \mathbf{b} \\
& \times[d(k-\alpha)-d(k-\alpha-1)+d(k-\alpha+1)-d(k-\alpha)] \\
& +\cdots+\mathbf{A b} \\
& \times \mathbf{b}[d(k-\alpha)-d(k-\alpha-1)+\cdots+d(k)-d(k-1)] .
\end{aligned}
$$

Let us introduce the following notation:

$\mathbf{R}(k)$

$$
\begin{aligned}
& =\mathbf{A}^{\alpha} \mathbf{b}[d(k-\alpha)-d(k-\alpha-1)] \\
& +\mathbf{A}^{\alpha-1} \mathbf{b} \\
& \quad \times[d(k-\alpha)-d(k-\alpha-1)+d(k-\alpha+1)-d(k-\alpha)] \\
& +\cdots+\mathbf{A b} \\
& \quad \quad \times[d(k-\alpha)-d(k-\alpha-1)+\cdots+d(k-1) \\
& \quad \quad-d(k-2)] \\
& +\mathbf{b}[d(k-\alpha)-d(k-\alpha-1)+\cdots+d(k)-d(k-1)] .
\end{aligned}
$$

We substitute $\mathbf{v}_{1}(k)$ from (9) and $\mathbf{R}(k)$ from (19) into (18) and obtain

$$
\begin{aligned}
\mathbf{x}(k+1)= & \mathbf{A}^{k+1} \mathbf{x}(0)+\sum_{m=0}^{k} \mathbf{A}^{k-m} \mathbf{b} u(m) \\
& +\sum_{m=0}^{k-\alpha-1} \mathbf{A}^{m} \mathbf{b} d(k-\alpha-1-m) \\
& +\mathbf{A}^{k-\alpha} \mathbf{v}_{1}(\alpha+1)+\mathbf{A}^{k-\alpha-1} \mathbf{v}_{1}(\alpha+2)
\end{aligned}
$$

$$
\begin{aligned}
& +\cdots+\mathbf{A}^{n} \mathbf{v}_{1}(k-n+1) \\
& +\mathbf{A}^{n-1} \mathbf{v}_{1}(k-n+2)+\cdots+\mathbf{A}^{2} \mathbf{v}_{1}(k-1) \\
& +\mathbf{A v}_{1}(k)+\mathbf{R}(k) .
\end{aligned}
$$

Then, we substitute $u(k)$ from (14) into (20) and get

$$
\begin{aligned}
\mathbf{x}(k+1)= & \mathbf{A}^{k+1} \mathbf{x}(0)+\sum_{m=0}^{k} \mathbf{A}^{k-m} \mathbf{b} u_{0}(m) \\
& +\sum_{m=0}^{k} \mathbf{A}^{k-m} \mathbf{b} u_{1}(m)+\cdots+\sum_{m=0}^{k} \mathbf{A}^{k-m} \mathbf{b} u_{n}(m) \\
& -\sum_{m=0}^{k} \mathbf{A}^{k-m} \mathbf{b}\left(\mathbf{c}^{\mathbf{T}} \mathbf{b}\right)^{-1} \mathbf{c}^{\mathrm{T}} \mathbf{w}(m) \\
& +\sum_{m=0}^{k-\alpha-1} \mathbf{A}^{m} \mathbf{b} d(k-\alpha-1-m) \\
& +\mathbf{A}^{k-\alpha} \mathbf{v}_{1}(\alpha+1)+\mathbf{A}^{k-\alpha-1} \mathbf{v}_{1}(\alpha+2) \\
& +\cdots+\mathbf{A}^{n} \mathbf{v}_{1}(k-n+1) \\
& +\mathbf{A}^{n-1} \mathbf{v}_{1}(k-n+2)+\cdots+\mathbf{A}^{2} \mathbf{v}_{1}(k-1) \\
& +\mathbf{A v}_{1}(k)+\mathbf{R}(k) .
\end{aligned}
$$

Relation (8) gives $\mathbf{w}(k)=\mathbf{0}$ for any $k \leq \alpha$. Therefore, from (9), we know that $\mathbf{v}_{1}(k)=\mathbf{0}$ for all $k \leq \alpha$ and consequently $\mathbf{v}_{i}(k)=\mathbf{0}$ for any $k<\alpha+i$. From (10) we know that $u_{i}(k)$ is a function of $\mathbf{v}_{i}(k)$, which gives $u_{i}(k)=0$ for $k<\alpha+i$. Taking that into consideration, we rewrite (21) as

$$
\begin{aligned}
\mathbf{x}(k+1)= & \mathbf{A}^{k+1} \mathbf{x}(0)+\sum_{m=0}^{k} \mathbf{A}^{k-m} \mathbf{b} u_{0}(m) \\
& +\sum_{m=\alpha+1}^{k} \mathbf{A}^{k-m} \mathbf{b} u_{1}(m) \\
& +\cdots+\sum_{m=\alpha+n}^{k} \mathbf{A}^{k-m} \mathbf{b} u_{n}(m) \\
& -\sum_{m=\alpha+1}^{k} \mathbf{A}^{k-m} \mathbf{b}\left(\mathbf{c}^{\mathrm{T}} \mathbf{b}\right)^{-1} \mathbf{c}^{\mathrm{T}} \mathbf{w}(m) \\
& +\sum_{m=0}^{k-\alpha-1} \mathbf{A}^{m} \mathbf{b} d(k-\alpha-1-m) \\
& +\mathbf{A}^{k-\alpha} \mathbf{v}_{1}(\alpha+1)+\mathbf{A}^{k-\alpha-1} \mathbf{v}_{1}(\alpha+2) \\
& +\cdots+\mathbf{A}^{n} \mathbf{v}_{1}(k-n+1)+\mathbf{A}^{n-1} \mathbf{v}_{1}(k-n+2) \\
& +\cdots+\mathbf{A}^{2} \mathbf{v}_{1}(k-1)+\mathbf{A} \mathbf{v}_{1}(k)+\mathbf{R}(k) .
\end{aligned}
$$


It can be seen from (3) that

$$
\begin{aligned}
\mathbf{A}^{k+1} \mathbf{x}(0)+\sum_{m=0}^{k} \mathbf{A}^{k-m} \mathbf{b} u_{0}(m) \\
=\mathbf{A}^{k+1} \mathbf{z}(0)+\sum_{m=0}^{k} \mathbf{A}^{k-m} \mathbf{b} u_{0}(m)=\mathbf{z}(k+1) .
\end{aligned}
$$

Moreover, from (8), we know that

$$
\begin{aligned}
& -\sum_{m=\alpha+1}^{k} \mathbf{A}^{k-m} \mathbf{b}\left(\mathbf{c}^{\mathrm{T}} \mathbf{b}\right)^{-1} \mathbf{c}^{\mathbf{T}} \mathbf{w}(m)+\sum_{m=0}^{k-\alpha-1} \mathbf{A}^{m} \mathbf{b} d(k-\alpha-1-m) \\
& =-\sum_{m=\alpha+1}^{k} \mathbf{A}^{k-m} \mathbf{b} d(m-\alpha-1) \\
& \quad+\sum_{m=0}^{k-\alpha-1} \mathbf{A}^{m} \mathbf{b} d(k-\alpha-1-m) \\
& =-\sum_{m=0}^{k-\alpha-1} \mathbf{A}^{m} \mathbf{b} d(k-m-\alpha-1) \\
& \quad+\sum_{m=0}^{k-\alpha-1} \mathbf{A}^{m} \mathbf{b} d(k-\alpha-1-m)=0 .
\end{aligned}
$$

Taking into consideration (23) and (24), we rewrite (22) as

$$
\begin{aligned}
\mathbf{x}(k+1)= & \mathbf{z}(k+1)+\sum_{m=\alpha+1}^{k} \mathbf{A}^{k-m} \mathbf{b} u_{1}(m) \\
& +\cdots+\sum_{m=\alpha+n}^{k} \mathbf{A}^{k-m} \mathbf{b} u_{n}(m) \\
& +\mathbf{A}^{k-\alpha} \mathbf{v}_{1}(\alpha+1)+\mathbf{A}^{k-\alpha-1} \mathbf{v}_{1}(\alpha+2) \\
& +\cdots+\mathbf{A}^{n} \mathbf{v}_{1}(k-n+1)+\mathbf{A}^{n-1} \mathbf{v}_{1}(k-n+2) \\
& +\cdots+\mathbf{A}^{2} \mathbf{v}_{1}(k-1)+\mathbf{A v}_{1}(k)+\mathbf{R}(k)
\end{aligned}
$$

We rearrange the elements of (25) as follows:

$$
\begin{aligned}
\mathbf{x}(k+1) & \\
= & \mathbf{z}(k+1)+\mathbf{A}^{k-\alpha} \mathbf{v}_{1}(\alpha+1)+\sum_{i=1}^{n} \mathbf{A}^{k-\alpha-i} \mathbf{b} u_{i}(\alpha+i) \\
& +\mathbf{A}^{k-\alpha-1} \mathbf{v}_{1}(\alpha+2)+\sum_{i=1}^{n} \mathbf{A}^{k-\alpha-i-1} \mathbf{b} u_{i}(\alpha+i+1) \\
& +\cdots+\mathbf{A}^{n} \mathbf{v}_{1}(k-n+1)+\sum_{i=1}^{n} \mathbf{A}^{n-i} \mathbf{b} u_{i}(k-n+i)
\end{aligned}
$$

$$
\begin{aligned}
& +\mathbf{A}^{n-1} \mathbf{v}_{1}(k-n+2)+\sum_{i=1}^{n-1} \mathbf{A}^{n-1-i} \mathbf{b} u_{i}(k-n+1+i) \\
& +\cdots+\mathbf{A}^{2} \mathbf{v}_{1}(k-1)+\sum_{i=1}^{2} \mathbf{A}^{2-i} \mathbf{b} u_{i}(k-2+i) \\
& +\mathbf{A} \mathbf{v}_{1}(k)+\mathbf{b} u_{1}(k)+\mathbf{R}(k) .
\end{aligned}
$$

Using (11), we simplify (26) in the following way:

$$
\begin{aligned}
\mathbf{x}(k+1) & \\
= & \mathbf{z}(k+1)+\mathbf{A}^{k-n-\alpha} \mathbf{v}_{n+1}(\alpha+n+1) \\
& +\mathbf{A}^{k-n-\alpha-1} \mathbf{v}_{n+1}(\alpha+n+2)+\cdots+\mathbf{v}_{n+1}(k+1) \\
& +\mathbf{v}_{n}(k+1)+\cdots+\mathbf{v}_{3}(k+1)+\mathbf{v}_{2}(k+1)+\mathbf{R}(k) .
\end{aligned}
$$

From Lemma 1, we know that for each $k$ vector $\mathbf{v}_{n+1}(k)=\mathbf{0}$, which gives us

$$
\begin{aligned}
\mathbf{x}(k+1)= & \mathbf{z}(k+1)+\mathbf{v}_{n}(k+1)+\cdots+\mathbf{v}_{3}(k+1) \\
& +\mathbf{v}_{2}(k+1)+\mathbf{R}(k) .
\end{aligned}
$$

The product $\mathbf{c}^{\mathbf{T}} \mathbf{x}$ can therefore be expressed as

$$
\begin{aligned}
\mathbf{c}^{\mathrm{T}} \mathbf{x}(k+1)= & \mathbf{c}^{\mathrm{T}} \mathbf{z}(k+1) \mathbf{c}^{\mathrm{T}} \mathbf{v}_{n}(k+1)+\cdots+\mathbf{c}^{\mathrm{T}} \mathbf{v}_{3}(k+1) \\
& +\mathbf{c}^{\mathrm{T}} \mathbf{v}_{2}(k+1)+\mathbf{c}^{\mathrm{T}} \mathbf{R}(k) .
\end{aligned}
$$

Lemma 1 states that, for each $k$ and $i=2, \ldots, n$, the product $\mathbf{c}^{\mathbf{T}} \mathbf{v}_{i}(k)=0$, which results in

$$
\mathbf{c}^{\mathrm{T}} \mathbf{x}(k+1)=\mathbf{c}^{\mathrm{T}} \mathbf{z}(k+1)+\mathbf{c}^{\mathrm{T}} \mathbf{R}(k) .
$$

From (12), we get

$$
\mathbf{c}^{\mathrm{T}} \mathbf{z}(k+1)=\mathbf{c}^{\mathrm{T}} \mathbf{x}_{d}(k+1)
$$

for $k>k^{*}$, which gives us

$$
\mathbf{c}^{\mathrm{T}} \mathbf{x}(k+1)=\mathbf{c}^{\mathrm{T}} \mathbf{x}_{d}(k+1)+\mathbf{c}^{\mathrm{T}} \mathbf{R}(k)
$$

and consequently

$$
s(k)=c^{\mathrm{T}} \mathbf{x}_{d}(k+1)-\mathbf{c}^{\mathrm{T}} \mathbf{x}(k+1)=-\mathbf{c}^{\mathrm{T}} \mathbf{R}(k) .
$$


Therefore, the bound of the absolute value of $|s(k)|$ can be expressed as

$$
\begin{aligned}
& \left|\mathbf{c}^{\mathbf{T}} \mathbf{R}(k)\right| \\
& =\mid \mathbf{c}^{\mathrm{T}} \mathbf{A}^{\alpha} \mathbf{b}[d(k-\alpha)-d(k-\alpha-1)] \\
& +\mathbf{c}^{\mathbf{T}} \mathbf{A}^{\alpha-1} \mathbf{b}[d(k-\alpha)-d(k-\alpha-1) \\
& +d(k-\alpha+1)-d(k-\alpha)] \\
& +\cdots+\mathbf{c}^{\mathrm{T}} \mathbf{A b}[d(k-\alpha)-d(k-\alpha-1) \\
& +\cdots+d(k-1)-d(k-2)] \\
& +\mathbf{c}^{\mathrm{T}} \mathbf{b}[d(k-\alpha)-d(k-\alpha-1) \\
& +\cdots+d(k)-d(k-1)] \\
& \leq\left|\mathbf{c}^{\mathrm{T}} \mathbf{A}^{\alpha} \mathbf{b}\right| \cdot|d(k-\alpha)-d(k-\alpha-1)| \\
& +\left|\mathbf{c}^{\mathrm{T}} \mathbf{A}^{\alpha-1} \mathbf{b}\right| \cdot \mid d(k-\alpha)-d(k-\alpha-1) \\
& +d(k-\alpha+1)-d(k-\alpha) \mid \\
& +\cdots+\left|\mathbf{c}^{\mathrm{T}} \mathrm{Ab}\right| \\
& \cdot \mid d(k-\alpha)-d(k-\alpha-1)+\cdots+d(k-1) \\
& -d(k-2) \mid \\
& +\left|\mathbf{c}^{\mathrm{T}} \mathbf{b}\right| \\
& \cdot|d(k-\alpha)-d(k-\alpha-1)+\cdots+d(k)-d(k-1)| \\
& \leq\left|\mathbf{c}^{\mathrm{T}} \mathbf{A}^{\alpha} \mathbf{b}\right| \cdot|d(k-\alpha)-d(k-\alpha-1)| \\
& +\left|\mathbf{c}^{\mathrm{T}} \mathbf{A}^{\alpha-1} \mathbf{b}\right| \cdot[|d(k-\alpha)-d(k-\alpha-1)| \\
& +|d(k-\alpha+1)-d(k-\alpha)|] \\
& +\cdots+\left|\mathbf{c}^{\mathrm{T}} \mathrm{Ab}\right| \\
& \cdot[|d(k-\alpha)-d(k-\alpha-1)| \\
& +\cdots+|d(k-1)-d(k-2)|] \\
& +\left|\mathbf{c}^{\mathrm{T}} \mathbf{b}\right| \cdot[|d(k-\alpha)-d(k-\alpha-1)| \\
& +\cdots+|d(k)-d(k-1)|] \\
& \leq\left|\mathbf{c}^{\mathrm{T}} \mathbf{A}^{\alpha} \mathbf{b}\right| \cdot \Delta_{\max }+\left|\mathbf{c}^{\mathrm{T}} \mathbf{A}^{\alpha-1} \mathbf{b}\right| \cdot 2 \Delta_{\max } \\
& +\cdots+\left|\mathbf{c}^{\mathrm{T}} \mathbf{A b}\right| \cdot \alpha \Delta_{\max }+\left|\mathbf{c}^{\mathrm{T}} \mathbf{b}\right| \cdot(\alpha+1) \Delta_{\max } \\
& =\sum_{i=0}^{\alpha}\left|\mathbf{c}^{\mathrm{T}} \mathbf{A}^{\alpha-i} \mathbf{b}\right| \cdot(i+1) \cdot \Delta_{\max } .
\end{aligned}
$$

We conclude that (15) holds for any $k>\max \left\{\alpha+n, k^{*}\right\}$.

We have shown that, upon applying control strategy (14) to the plant, the system state is driven into a certain band around the sliding hyperplane in finite time and contained within it in all subsequent time instants. As specified by Theorem 2, the width of the band can be expressed by (15).

\section{Simulation Example}

We will now show the effectiveness of the proposed method by means of a simulation example. The control law proposed in this paper will be applied to the following linear plant:

$$
\mathbf{A}=\left[\begin{array}{ccc}
1 & 0.5 & 0 \\
0 & 1 & 1 \\
0 & 0 & 1
\end{array}\right] \quad \mathbf{b}=\left[\begin{array}{l}
0 \\
0 \\
1
\end{array}\right] \quad \mathbf{x}(0)=\left[\begin{array}{l}
10 \\
10 \\
10
\end{array}\right]
$$

Our objective is to drive the system output $\mathbf{x}_{1}(k)$ along a desired trajectory described as $25 \sin (k \pi / 50)$. It can be seen from (35) that the desired state vector $\mathbf{x}_{d}(k)$ can be expressed as

$$
\begin{aligned}
& \mathbf{x}_{d}(k) \\
& =\left[\begin{array}{c}
25 \sin \left(\frac{k \pi}{50}\right) \\
50 \sin \left(\frac{(k+1) \pi}{50}\right)-50 \sin \left(\frac{k \pi}{50}\right) \\
50 \sin \left(\frac{(k+2) \pi}{50}\right)-100 \sin \left(\frac{(k+1) \pi}{50}\right)+50 \sin \left(\frac{k \pi}{50}\right)
\end{array}\right] .
\end{aligned}
$$

The system is subject to state measurement delay $\alpha=3$. Vector $\mathbf{c}$ for the control law (14) is chosen according to (5) and $c^{\mathrm{T}}=\left[\begin{array}{lll}2 & 2 & 1\end{array}\right]$. We select the parameter $k^{*}=5$ to ensure that the sliding variable rate of descent is not excessively big.

4.1. System Subject to Sinusoidal Disturbance. In the first example, we assume that system (35) is subject to matched disturbance

$$
d(k)=0.25 \cdot \sin \left(\frac{k \pi}{6}\right)
$$

Then, the maximum rate of change of the disturbance between any two subsequent time instants equals $\Delta_{\max }=$ 0.125 . Figure 1 illustrates the evolution of the sliding variable $s(k)$ upon applying the control law (14). According to (15), the bounds of the quasi-sliding mode band equal \pm 4.375 , and it can actually be seen from the figure that the bounds are not exceeded. Figure 2 shows system output $x_{1}(k)$. The black dashed line in this figure illustrates the desired value of the output $x_{d 1}(k)$. Figure 3 illustrates the control signal.

It can be seen from Figure 1 that the sliding variable arrives inside the quasi-sliding mode band specified in Theorem 2 in a finite amount of steps not greater than $k^{*}+1=6$ and is contained within it for all subsequent time instants. Moreover, as seen from Figure 2, the proposed control strategy effectively realizes tracking of the system output along the desired trajectory despite the detrimental effects of disturbance and measurement delay. 


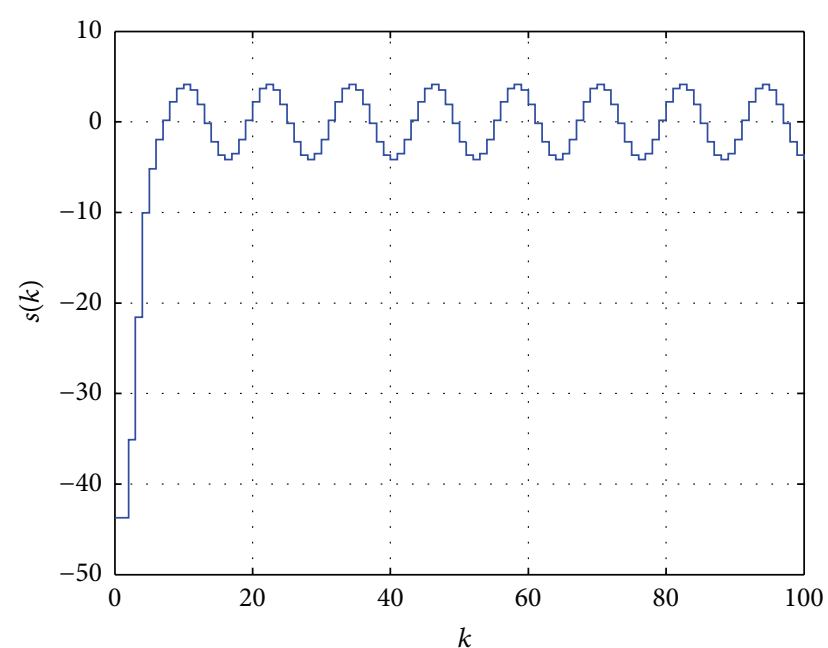

FIGURE 1: Sliding variable $s$.

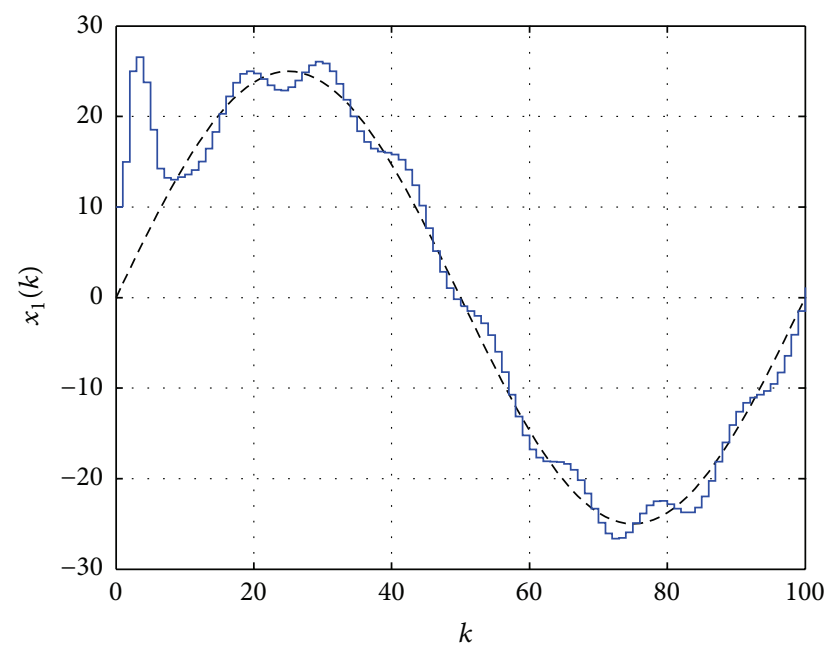

FIGURE 2: System output $x_{1}$.

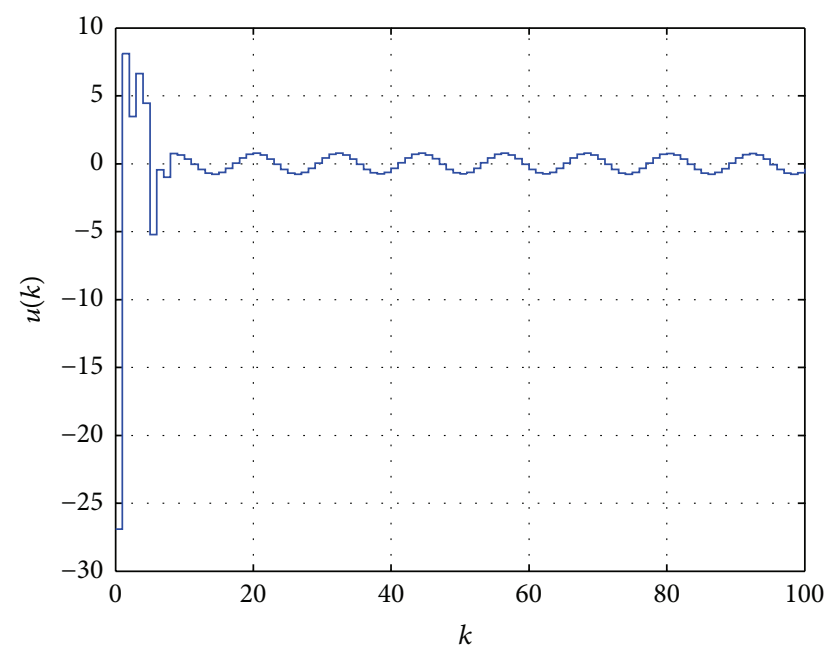

Figure 3: Control signal $u$.
TABLE 1: IAE and ISE for $k=0, \ldots, 100$

\begin{tabular}{lcc}
\hline & IAE & ISE \\
\hline Sliding variable $s$ & 406.2 & 6467.1 \\
System output $x_{1}$ & 222.6 & 1889.5 \\
\hline
\end{tabular}

In order to assess the control quality obtained in this simulation example, we demonstrate the deviation of sliding variable $s$ from 0 and system output $x_{1}$ from $x_{d 1}$ using two control quality criteria: integral absolute error (IAE) and integral squared error (ISE). The criteria are shown in Table 1.

4.2. System Subject to Unbounded Disturbance. The first simulation example has shown that stability of the system as well as tracking of the system desired output is successfully obtained in the presence of slowly changing disturbance. However, disturbance (37) is not only characterized by a limited rate of change, but its magnitude is also limited. Therefore, in the second simulation example, the considered system will be subject to unbounded disturbance with limited rate of change and it will be demonstrated that the advantageous properties shown in the previous example are preserved. The disturbance affecting the plant in the second example can be expressed as

$$
\begin{aligned}
d(k)= & 0.125 \\
& \cdot\left\{\text { floor }\left[\frac{k}{10}\right]+\text { floor }\left[\frac{(k+1)}{10}\right]+\text { floor }\left[\frac{(k+2)}{10}\right]\right\} .
\end{aligned}
$$

It can be seen that the magnitude of disturbance (38) is unbounded and its rate of change is limited by $\Delta_{\max }=0.125$. Therefore, the bounds of the quasi-sliding mode band again equal \pm 4.375 . Figure 4 shows the evolution of the sliding variable, Figure 5 illustrates the system output (once again, the desired trajectory is illustrated by a black dashed line), and Figure 6 shows the control signal.

It can be seen from Figure 4 that the sliding variable arrives inside the quasi-sliding mode band in finite time and remains within it in all subsequent time instants. Figure 5 shows that tracking of the demand system output is realized in the presence of unbounded disturbance. Like in the previous example, we demonstrate the deviation of the sliding variable from 0 and system output from $x_{d 1}$ using IAE and ISE. The criteria are given in Table 2.

\section{Conclusions}

In this paper, the problem of sliding mode control of a discrete-time system with state measurement delay has been explored. In order to ensure that the unpredictable disturbance is effectively counteracted in an environment with time delay, we have designed a novel sliding mode controller for such systems. The proposed control method utilizes two models of the considered plant. The first one is responsible for retrieving information about disturbance affecting the plant and reducing its influence on the system state to zero 


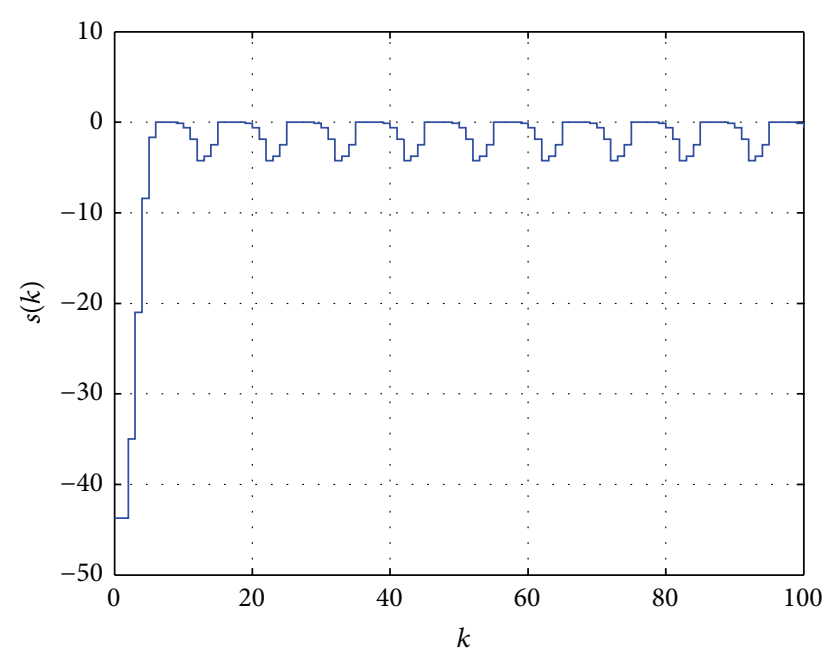

FIGURE 4: Sliding variable $s$.

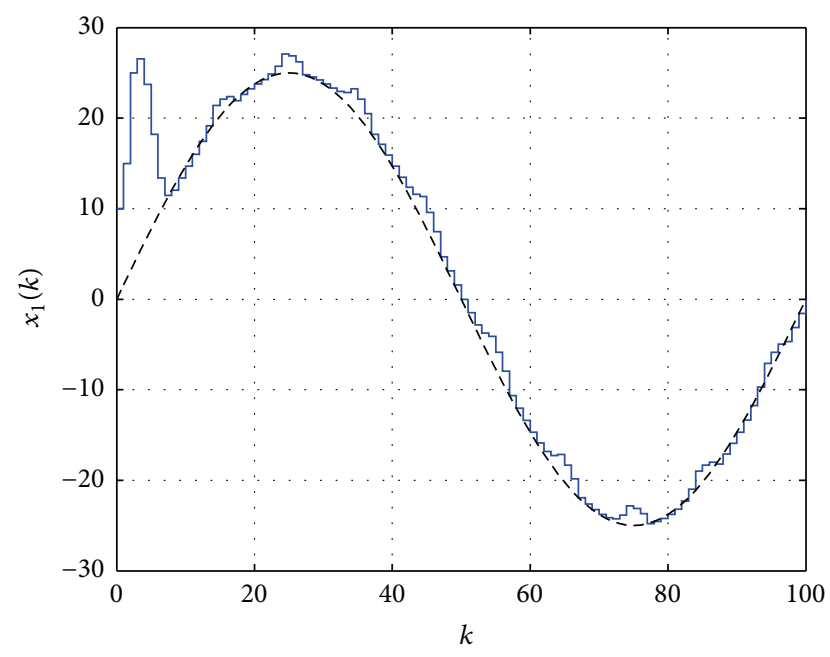

Figure 5: System output $x_{1}$.

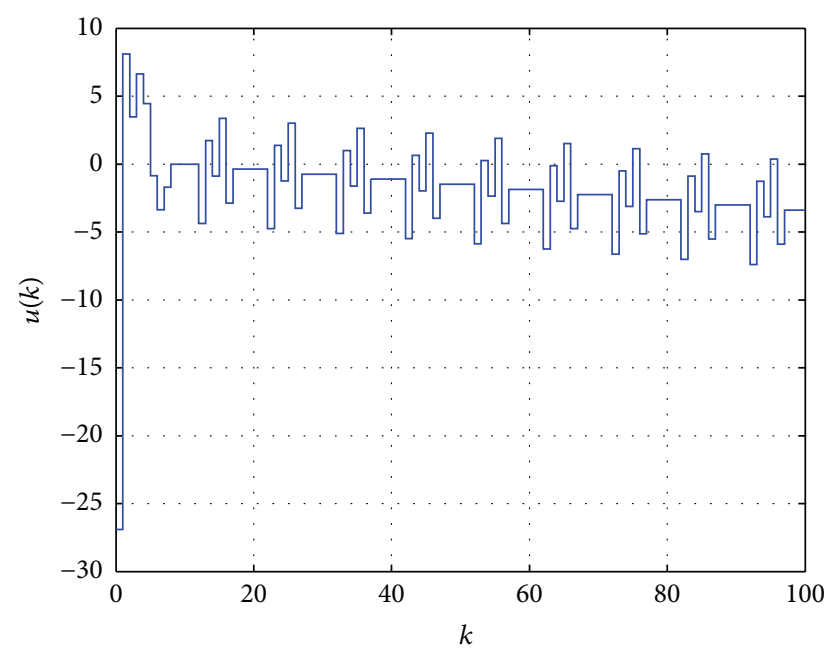

Figure 6: Control signal $u$.
TABLE 2: IAE and ISE for $k=0, \ldots, 100$.

\begin{tabular}{lcc}
\hline & IAE & ISE \\
\hline Sliding variable $s$ & 272.4 & 5944.3 \\
System output $x_{1}$ & 159.3 & 1766.4 \\
\hline
\end{tabular}

in finite time. The second model drives the system state along a desired trajectory $\mathbf{x}_{d}(k)$. The result is obtained under the assumption that the disturbance affecting the plant has an upper bounded rate of change, but the boundedness of the disturbance itself is not required. It has been proven that the new controller confines the system state to a given band around the sliding hyperplane and the effectiveness of the proposed method has been verified with simulation examples.

\section{Conflict of Interests}

The authors declare that there is no conflict of interests regarding the publication of this paper.

\section{Acknowledgments}

This work has been performed in the framework of the project "Optimal Sliding Mode Control of Time Delay Systems" financed by the National Science Centre of Polanddecision no. DEC 2011/01/B/ST7/02582. Kind support provided by the Foundation for Polish Science under "Mistrz" Grant is also acknowledged.

\section{References}

[1] S. V. Emelyanov, Variable Structure Control Systems, Nauka, Moscow, Russia, 1967, (Russian).

[2] V. I. Utkin, "Variable structure systems with sliding modes," IEEE Transactions on Automatic Control, vol. 22, no. 2, pp. 212$222,1977$.

[3] B. Draženović, "The invariance conditions in variable structure systems," Automatica, vol. 5, pp. 287-295, 1969.

[4] A. Bartoszewicz and A. Nowacka-Leverton, Time-Varying Sliding Modes for Second and Third Order Systems, vol. 382 of Lecture Notes in Control and Information Sciences, Springer, Berlin Heidelberg, Germany, 2009.

[5] R. A. DeCarlo, S. H. Zak, and G. P. Matthews, "Variable structure control of nonlinear multivariable systems: a tutorial," Proceedings of the IEEE, vol. 76, no. 3, pp. 212-232, 1988.

[6] C. Edwards and S. Spurgeon, Sliding Mode Control: Theory and Applications, Taylor \& Francis, London, UK, 1998.

[7] W. Gao and J. C. Hung, "Variable structure control of nonlinear systems: a new approach," IEEE Transactions on Industrial Electronics, vol. 40, no. 1, pp. 45-55, 1993.

[8] V. Utkin, J. Guldner, and J. Shi, Sliding Mode Control in ElectroMechanical Systems, Taylor \& Francis, 2nd edition, 2009.

[9] C. Milosavljevic, "General conditions for the existence of a quasisliding mode on the switching hyperplane in discrete variable structure systems," Automation and Remote Control, vol. 46, no. 3, pp. 307-314, 1985. 
[10] V. Utkin and S. V. Drakunov, "On discrete-time sliding mode control," in Proceedings of the IFAC Conference on Nonlinear Control, pp. 484-489, 1989.

[11] B. Bandyopadhyay and D. Fulwani, "High-performance tracking controller for discrete plant using nonlinear sliding surface," IEEE Transactions on Industrial Electronics, vol. 56, no. 9, pp. 3628-3637, 2009.

[12] B. Bandyopadhyay and S. Janardhanan, Discrete-Time Sliding Mode Control. A Multirate Output Feedback Approach, Springer, Berlin, Germany, 2006.

[13] G. Bartolini, A. Ferrara, and V. I. Utkin, "Adaptive sliding mode control in discrete-time systems," Automatica, vol. 31, no. 5, pp. 769-773, 1995.

[14] A. Bartoszewicz, "Remarks on "discrete-time variable structure control systems',' IEEE Transactions on Industrial Electronics, vol. 43 , no. 1, pp. 235-238, 1996.

[15] A. Bartoszewicz, "Discrete-time quasi-sliding-mode control strategies," IEEE Transactions on Industrial Electronics, vol. 45, no. 4, pp. 633-637, 1998.

[16] A. Bartoszewicz and J. Żuk, "Discrete time sliding mode flow controller for multi-source single-bottleneck connectionoriented communication networks," Journal of Vibration and Control, vol. 15, no. 11, pp. 1745-1760, 2009.

[17] S. Chakrabarty and B. Bandyopadhyay, "Quasi sliding mode control with quantization in state measurement," in Proceedings of the 37th Annual Conference of the IEEE Industrial Electronics Society, pp. 3971-3976, November 2011.

[18] M. L. Corradini and G. Orlando, "Variable structure control of discretized continuous-time systems," IEEE Transactions on Automatic Control, vol. 43, no. 9, pp. 1329-1334, 1998.

[19] M. L. Corradini, V. Fossi, A. Giantomassi, G. Ippoliti, S. Longhi, and G. Orlando, "Discrete time sliding mode control of robotic manipulators: development and experimental validation," Control Engineering Practice, vol. 20, no. 8, pp. 816-822, 2012.

[20] K. Furuta, "Sliding mode control of a discrete system," Systems and Control Letters, vol. 14, no. 2, pp. 145-152, 1990.

[21] W. Gao, Y. Wang, and A. Homaifa, "Discrete-time variable structure control systems," IEEE Transactions on Industrial Electronics, vol. 42, no. 2, pp. 117-122, 1995.

[22] G. Golo and C. Milosavljević, "Robust discrete-time chattering free sliding mode control," Systems and Control Letters, vol. 41, no. 1, pp. 19-28, 2000.

[23] S. Janardhanan and B. Bandyopadhyay, "Output feedback sliding-mode control for uncertain systems using fast output sampling technique," IEEE Transactions on Industrial Electronics, vol. 53, no. 5, pp. 1677-1682, 2006.

[24] S. Janardhanan and B. Bandyopadhyay, "Multirate output feedback based robust quasi-sliding mode control of discrete-time systems," IEEE Transactions on Automatic Control, vol. 52, no. 3, pp. 499-503, 2007.

[25] S. Janardhanan and V. Kariwala, "Multirate-output-feedbackbased LQ-optimal discrete-time sliding mode control," IEEE Transactions on Automatic Control, vol. 53, no. 1, pp. 367-373, 2008.

[26] S. Kurode, B. Bandyopadhyaya, and P. S. Gandhi, "Discrete sliding mode control for a class of underactuated systems," in Proceedings of the 37th Annual Conference of the IEEE Industrial Electronics Society, pp. 3936-3941, November 2011.

[27] A. Mehta and B. Bandyopadhyay, "Frequency-shaped sliding mode control using output sampled measurements," IEEE Transactions on Industrial Electronics, vol. 56, no. 1, pp. 28-35, 2009.
[28] A. J. Mehta and B. Bandyopadhyay, "The design and implementation of output feedback based frequency shaped sliding mode controller for the smart structure," in Proceedings of the IEEE International Symposium on Industrial Electronics (ISIE '10), pp. 353-358, Bari, Italy, July 2010.

[29] S. J. Mija and S. Thomas, "Reaching law based sliding mode control for discrete MIMO systems," in Proceedings of the 11th International Conference on Control, Automation, Robotics and Vision (ICARCV'10), pp. 1291-1296, December 2010.

[30] Č. Milosavljević, B. Peruničić-Draženović, B. Veselić, and D. Mitić, "Sampled data quasi-sliding mode control strategies," in Proceedings of the IEEE International Conference on Industrial Technology, pp. 2640-2645, December 2006.

[31] Y. Niu, D. W. C. Ho, and Z. Wang, "Improved sliding mode control for discrete-time systems via reaching law," IET Control Theory and Applications, vol. 4, no. 11, pp. 2245-2251, 2010.

[32] Y. Pan and K. Furuta, "Variable structure control with sliding sector based on hybrid switching law," International Journal of Adaptive Control and Signal Processing, vol. 21, no. 8-9, pp. 764778, 2007.

[33] X. Yu, B. Wang, and X. Li, "Computer-controlled variable structure systems: the state-of-the-art," IEEE Transactions on Industrial Informatics, vol. 8, no. 2, pp. 197-205, 2012.

[34] K. J. Astrom and B. Wittenmark, Computer-Controlled Systems: Theory and Design, Prentice Hall, Upper Saddle River, NJ, USA, 1997.

[35] G. F. Franklin, J. D. Powell, and M. Workman, Digital Control of Dynamic Systems, Addison-Wesley, Longman Publishing, Reading, Mass, USA, 1997.

[36] W. Zhang, M. S. Branicky, and S. M. Phillips, "Stability of networked control systems," IEEE Control Systems Magazine, vol. 21, no. 1, pp. 84-97, 2001.

[37] W. P. Heemels, A. R. Teel, N. van de Wouw, and D. Nesic, "Networked control systems with communication constraints: tradeoffs between transmission intervals, delays and performance," IEEE Transactions on Automatic Control, vol. 55, no. 8, pp. 1781-1796, 2010.

[38] X. Luan, P. Shi, and F. Liu, "Stabilization of networked control systems with random delays," IEEE Transactions on Industrial Electronics, vol. 58, no. 9, pp. 4323-4330, 2011.

[39] R. S. Raji, "Smart networks for control," IEEE Spectrum, vol. 31, no. 6, pp. 49-55, 1994.

[40] Y. Tipsuwan and M.-Y. Chow, "Control methodologies in networked control systems," Control Engineering Practice, vol. 11, no. 10, pp. 1099-1111, 2003.

[41] G. C. Walsh, H. Ye, and L. Bushnell, "Stability analysis of networked control systems," IEEE Transactions on Control Systems Technology, vol. 10, no. 3, pp. 438-446, 2002.

[42] L. Dugard and E. I. Verriest, Stability and Control of Time-Delay Systems, Springer, Heidelberg, Germany, 1998.

[43] S. Janardhanan and B. Bandyopadhyay, "Output feedback discrete-time sliding mode control for time delay systems," IEE Proceedings on Control Theory and Applications, vol. 153, no. 4, pp. 387-396, 2006.

[44] A. J. Koshkouei and A. S. I. Zinober, "Sliding mode time-delay systems," in Proceedings of the IEEE International Workshop on Variable Structure Systems (VSS '96), pp. 97-101, December 1996.

[45] X. Q. Liu, W. Q. Ge, and Y. T. Chui, "Variable structure predictor controller with quasi sliding mode for systems with delay," in Proceedings of the IEEE International Symposium on Industrial Electronics, pp. 211-214, 1992. 
[46] Y. Xia, J. Han, and Y. Jia, "A sliding mode control for linear systems with input and state delays," in Proceedings of the 41st IEEE Conference on Decision and Control, pp. 3332-3337, Phoenix, Ariz, USA, December 2002.

[47] A. Bartoszewicz and P. Latosinski, "Quasi-sliding mode networked controller for linear discrete time systems subject to disturbance," in Proceedings of the 22nd Mediterranean Conference on Control and Automation, pp. 328-333, 2014. 


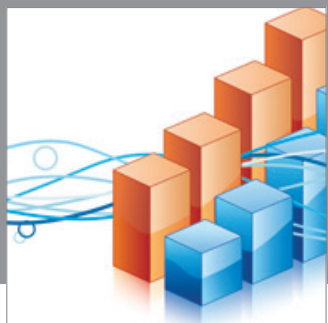

Advances in

Operations Research

mansans

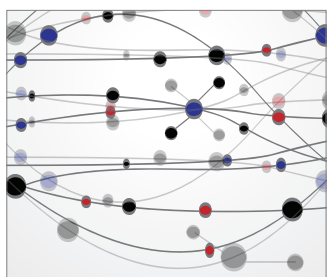

The Scientific World Journal
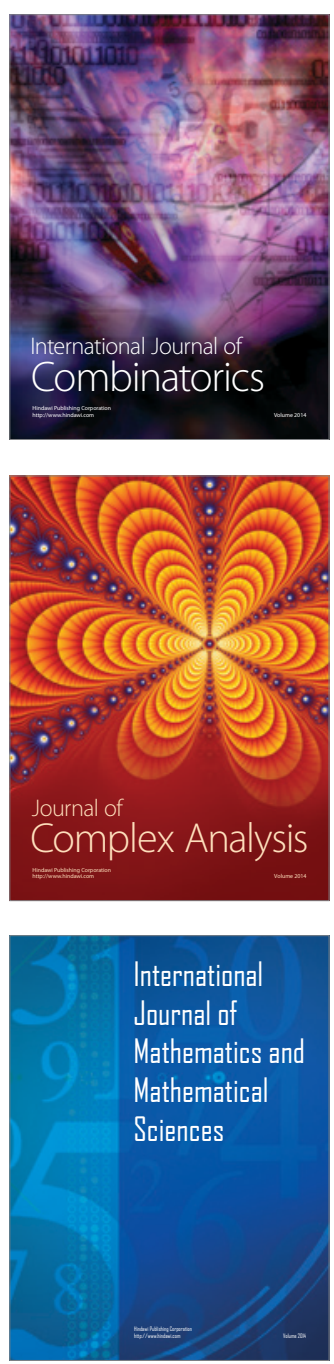
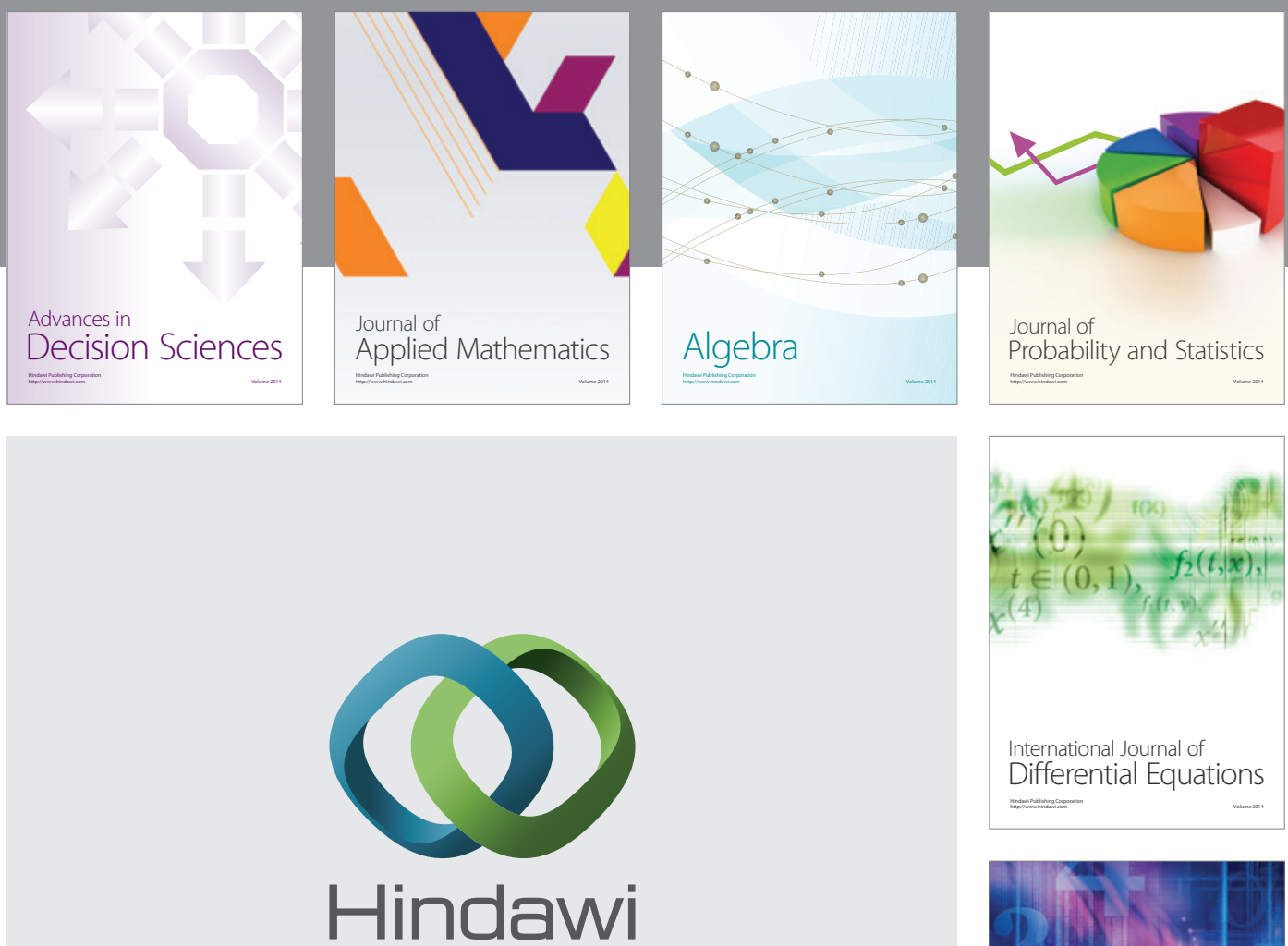

Submit your manuscripts at http://www.hindawi.com
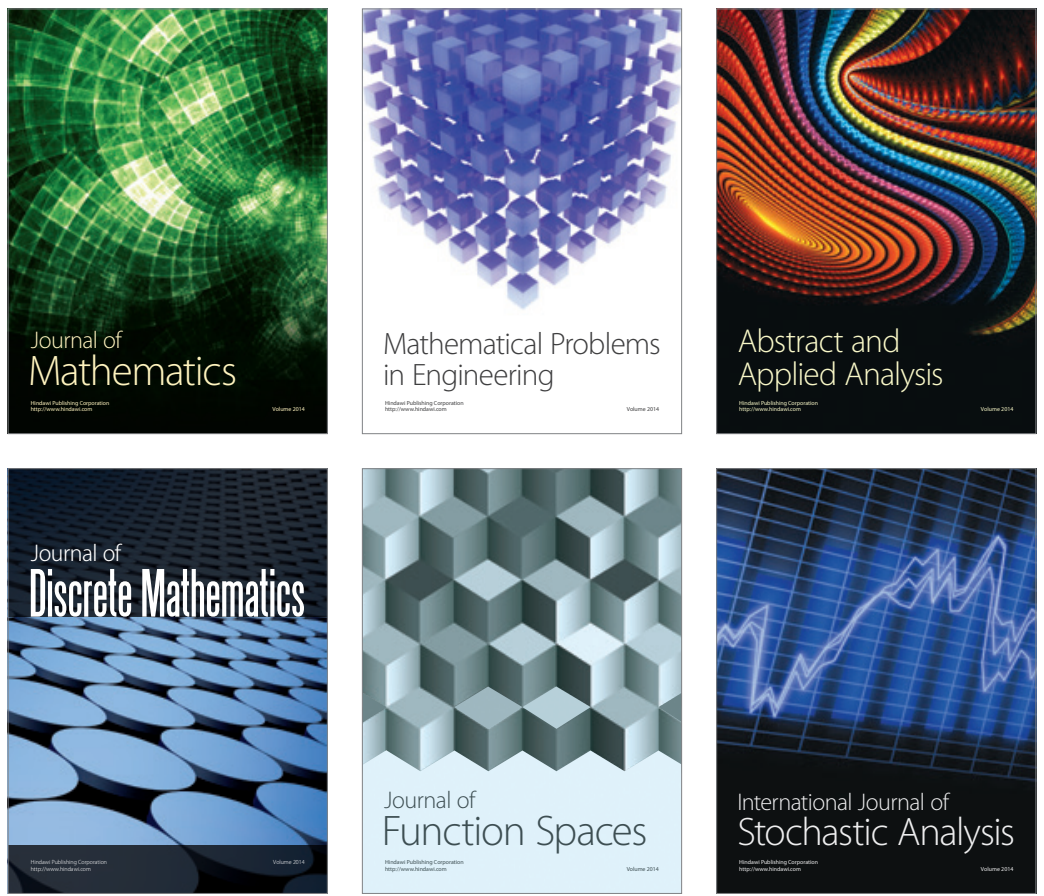

Journal of

Function Spaces

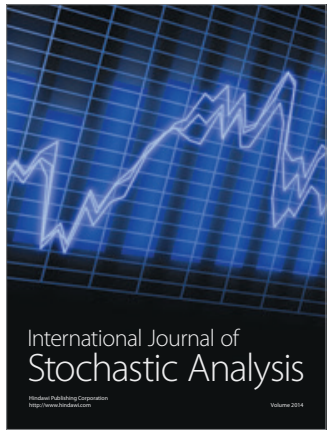

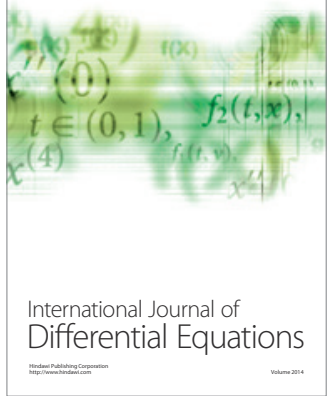
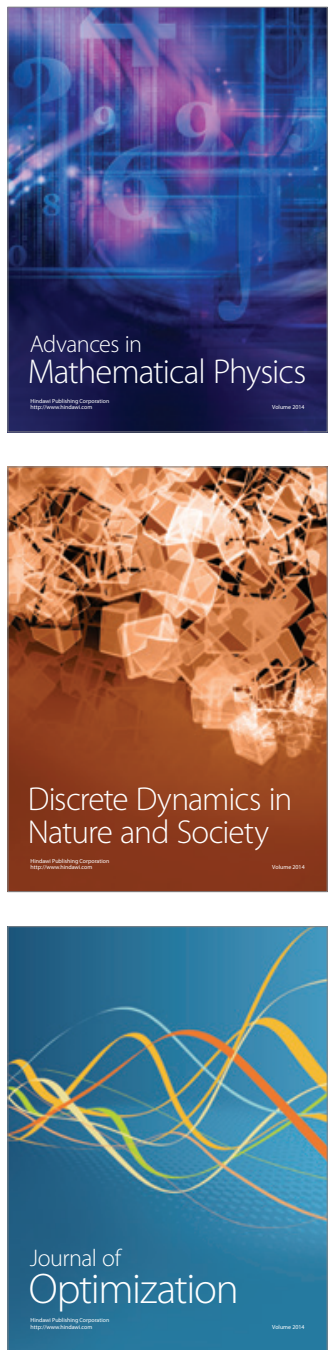